\title{
Design of Robust Fractional-Order PID Controller for DC Motor Using the Adjustable Performance Weights in the Weighted-Mixed Sensitivity Problem
}

\author{
Toufik Amieur ${ }^{1}$, Moussa Sedraoui ${ }^{2}$, Oualid Amieur ${ }^{3}$ \\ ${ }^{1}$ Department of Electrical Engineering, Kasdi Merbah University, Ouargla, Algeria \\ ${ }^{2,3}$ Telecommunication Laboratory, 8 May 1945 University, Guelma, Algeria
}

\section{Article Info \\ Article history: \\ Received Feb 2, 2018 \\ Revised Apr 15, 2018 \\ Accepted Apr 30, 2018}

\section{Keyword:}

Fractional-order PID controller FO-PID

Min-Max Optimization

Problem

Nominal Performance NP

Robust Stability RS

\begin{abstract}
This paper deals with the robust series and parallel fractional-order PID synthesis controllers with the automatic selection of the adjustable performance weights, which are given in the weighted-mixed sensitivity problem. The significant contribution of the paper is to achieve the good trade-off between nominal performances and robust stability for DC motor regardless its nonlinear dynamic behavior, the unstructured model uncertainties and the effect of the sensor noises on the feedback control system. The main goal is formulated as the weighted-mixed sensitivity problem with unknown adjustable performance weight. This problem is then solved using an adequate optimization algorithm and its optimal solution leads to determine simultaneously the robust fractional PID controller, which is proposed by the series and the parallel fractional structures, As well as, the obtained optimal solution determines the corresponding adjustable performance weight. The proposed control technique is applied on DC motor where its dynamic behavior is modeled by unstructured multiplicative model uncertainty. The obtained performances are compared in frequency- and time-domains with those given by both integer controllers such classical PID and $\mathrm{H} \infty$ controllers.
\end{abstract}

Copyright (C) 2018 Institute of Advanced Engineering and Science. All rights reserved.

\footnotetext{
Corresponding Author:

Toufik Amieur,

Department of Electrical Engineering,

Kasdi Merbah University,

Ouargla, Algeria.

Email: amieur.to@gmail.com
}

\section{INTRODUCTION}

Recently, one of the most desired aspects in $D C$ control motor is to achieve a good trade-off between $R S$ and NP of the feedback control system [1], [2]. Due to inaccurate modeling, component aging of mechanical part of DC motor, sensor noises, exterior conditions, and others, all proposed DC motor models unavoidably incorporate uncertainties and external disturbances.

In control engineering, the controller synthesis using the integer PID controller-structures is still widely recognized as one of the simplest yet most effective control strategies in industry [3-5]. However, the obtained $H_{\infty}$ performances analysis does not guarantee both $R S$ and $N P$, and optimal trade-off between them. Hence this trade-off should be enhanced when $D C$ motor is subjected to parametric uncertainties and measurement noises.

To avoid this problem, Matrix Inequality $L M I$ based $H_{\infty}$ control techniques or Algebraic Riccati equations AREs are usually preferred over other methods [6]-[7], due to its computational simplicity and efficiency. The controller parameters are designed from solving the weighted-mixed sensitivity problem where all the above-mentioned effects are presented using some weights in the weighted-mixed sensitivity 
problem. The obtained robust controller ensures good robustness properties. However, a significant shortcoming of these techniques leads to high-order controller. Its implementation leads to high cost, difficult commissioning, poor reliability, and potential problems in maintenance.

Recently, many researches have done $H_{\infty}$ control analysis of uncertain systems using fractional controller-structures, from which the controller parameters are optimized by an adequate optimization tool.

It may be noted that the fractional order carried-out on the Laplace operator is approximated by an equivalent integer transfer function, which contains the infinite poles and zeros [8]. Therefore, various advanced control design methods benefit to this property. Its controller is designed with the less number of the unknown parameters [8], [9]. It can also satisfy a high level of the imposed $H_{\infty}$ specifications where the model parameters change in wide range.

It is further noticed that the FO-PID controllers have been applied by researchers in different fields of engineering and industries around the world, such as in motion control of DC motor [9], [10], automatic voltage regulator [11], [12], aerospace designing control system [13], weapon system[14], wind energy system [15], nuclear reactor [16], hydropower turbine [17], and most of the above mentioned research results show that the FO-PID controller has a better performance and robustness than a conventional one. Although it is so, the parameter optimization of FO-PID controller is still an important and challenge issue until now.

In this paper, the robust FO-PID controller is proposed using both structures series and parallel. The objective is to optimize the controller parameters from solving the weighted-mixed problem, in which the adjustable performance weight is derived. The control strategy is applied on $D C$ motor to validate the efficiency of the prosed idea.

\section{ON THE FRACTIONAL CALCULUS}

Fractional calculus is a generalization of integration and differentiation of the non-integer order operator ${ }_{a} D_{t}^{\gamma}$, where $a$ and $t$ denote the limits of the operation [18]. The continuous integro-differential operator of order $\gamma$ is defined in the following way (1):

$$
{ }_{a} D_{t}^{\gamma}=\left\{\begin{array}{cc}
d^{\gamma} / d t^{\gamma} & : \gamma>0 \\
1 & : \gamma=0 \\
\int_{a}^{t}(d \tau)^{-\gamma} & : \gamma<0
\end{array}\right.
$$

So that the Riemann-Liouville definition is given as shown in (2).

$$
{ }_{a} D_{t}^{\gamma} f(t)=\frac{1}{\Gamma(n-\gamma)} \frac{d^{n}}{d t^{n}} \int_{a}^{t} \frac{f(\tau)}{(t-\tau)^{\gamma-n+1}} d \tau
$$

According to (2), $n$ denotes the integer part of $\gamma$ where $n-1<\gamma<n$ and $\Gamma$ (.) is the Euler's gamma function that given by $\Gamma(n)=\int_{0}^{+\infty} t^{n-1} e^{-n} d t$, where $\Gamma(n+1)=n !$. The Laplace transforms of the $R L$ fractional derivative/integral (2) under zero initial conditions for order $\gamma$ is given by (3):

$$
L\left({ }_{0} D_{t}^{ \pm \gamma} f(t)\right)=\int_{0}^{+\infty} e^{-s t}{ }_{0} D_{t}^{ \pm \gamma} f(t) d t=s^{ \pm \gamma} F(s)
$$

Noticing that, the implementation FO-PID controller needs to approximate its fractional part of powers $\gamma$ by the usual integer transfer functions with a similar behavior. The method is based on approximating $s^{\gamma}$ in a specified frequency range $\omega=\left[\omega_{h}, \omega_{b}\right]$ and of integer order $N$ by a rational transfer function obtained in the following manner (4) [19]:

$$
S^{\gamma}=C \prod_{k=-N}^{k=N} \frac{s+\omega_{k}^{\prime}}{S+\omega_{k}}
$$

From (4) the zeros, poles and gain are respectively defined as shown in (5-7). 


$$
\begin{aligned}
& \omega_{k}^{\prime}=\omega_{b}\left[\frac{\omega_{h}}{\omega_{b}}\right]^{\frac{k+N+\frac{1}{2}(1-\gamma)}{2 N+1}} \\
& \omega_{k}=\omega_{b}\left[\frac{\omega_{h}}{\omega_{b}}\right]^{\frac{k+N+\frac{1}{2}(1+\gamma)}{2 N+1}} \\
& C=\left[\frac{\omega_{h}}{\omega_{b}}\right]^{-\frac{\gamma}{2}} \prod_{k=-N}^{N}\left[\frac{\omega_{k}}{\omega_{k}^{\prime}}\right]
\end{aligned}
$$

In some fractional controller-structure, due to the commutative property of the fractional operator $s^{\alpha}$ and order $\alpha \geq 1$, it can be approximated by $s^{\alpha}=s^{n+\gamma}$ where $n=\alpha-\gamma$ is the integer part of $\alpha$ and $s^{\alpha}$ is approximated according to equation (4).

\section{ROBUST PARALLEL/SERIES FO-PID DESIGN CONTROLLER}

Some feedback control systems implement a FO-PID controller function on serial form, while others use the parallel form. The aim of this paper is to observe differences between them for the $D C$ motor, and to see the performances of each one in time and frequency domains.

\subsection{Robust Parallel FO-PID Controller}

The Robust parallel FO-PID controller called also PFO-PID is the general case of the classical parallel integer one. In time domain, the differential equation is defined by (8) [20]-[21]:

$$
u(t)=K_{p} * e(t)+K_{i} *{ }_{0} D_{t}^{-\lambda} e(t)+K_{d}{ }_{0} D_{t}^{+\mu} e(t)
$$

Where $e(t)$ and $u(t)$ are respectively, the set-error and the control signal. The transfer function of robust PFO-PID controller is therefore given through the following Laplace transform (90) [20].

$$
K(s, \underline{x})=K_{p}+K_{i} s^{-\lambda}+K_{d} s^{\mu}
$$

According to (9), the controller parameters are given by the design vector $\underline{x}=\left[K_{p}, K_{i}, K_{d}, \lambda, \mu\right]$ where its derivative part is usually replaced by the term $\frac{K_{d} s^{\mu}}{1+\tau S}$ in order to attenuate the noise amplification effect. We get (10):

$$
K(s, \underline{x})=K_{P}+K_{i} s^{-\lambda}+\frac{K_{d} s^{\mu}}{1+\tau s}
$$

So, there are six parameters to be tune, which are given by the design vector $\underline{x}=\left[K_{p}, K_{i}, K_{d}, \tau, \lambda, \mu\right]^{T}$.

\subsection{Robust Series FO-PID Controller}

The Robust series FO-PID controller called also SFO-PID becomes the general form the classical series integer PID controller. The differential equation is therefore given in time domain by (11) [22], [23]:

$$
u(t)=K_{p} * e(t)+K_{p} K_{i} *{ }_{0} D_{t}^{-\lambda} e(t)+K_{p} K_{d} *{ }_{0} D_{t}^{+\mu} e(t)+K_{p} K_{i} K_{d} *{ }_{0} D_{t}^{+\mu-\lambda} e(t)
$$


From (11) the transfer function is defined by (12), in which the pre-filter $\frac{1}{1+\tau \mathrm{s}}$ caun be included for the same previous reason, yields also (12):

$$
K(s, \underline{x})=K_{p} \cdot\left(1+K_{i} s^{-\lambda}\right) \cdot\left(1+\frac{K_{d} s^{\mu}}{1+\tau s}\right)
$$

where the weighted-mixed sensitivity problem is solved using the same prevois vector.

\section{WEIGHTED-MIXED SENSITIVITY FORMULATION PROBLEM}

Let consider the feedback control system shown in Figure 1 where $d_{y}(s)$ and $\eta(s)$ denote respectively, the load disturbance and the noise-measurements. Moreover, $r(s)$ and ${ }^{y(s)}$ are respectively, the set-point reference and the process output of the feedback control system.

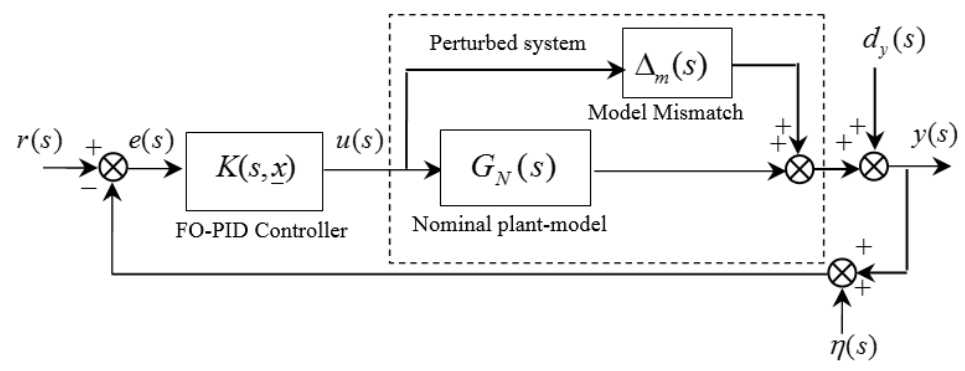

Figure 1. Block diagram of the feedback control system

Suppose that the nominal plant transfer function is $G_{N}(s)$ and consider the perturbed plant transfer function of the form $G_{P}(s)=\left[1+\Delta_{m}(s)\right] G_{N}(s)$. Here, $\Delta_{m}(s)$ is the normalized uncertainty that is assumed to satisfy (13):

$$
\left\|\Delta_{m}(s)\right\|_{\infty} \square \max _{\omega \in \square^{+}}\left\{\sigma_{\max }\left[\Delta_{m}(j \omega)\right]\right\} \leq 1
$$

where $\sigma_{\max }\left[\Delta_{m}(j \omega)\right]$ is the maximal singular value of $\Delta_{m}(s)$ at the frequency point $\omega$ and $\omega \in\left[\omega_{\min }, \omega_{\max }\right]$. In robust control theory the trade-off between $R S$ and $N P$ depends heavily by satisfying two following conditions, which are:

\subsection{RS Condition}

The robust FO-PID controller should guarantee the $R S$ that means the closed-loop system must remain stable in presence of all possible uncertainties. In order to secure the suitable $R S$, the complementary sensitivity transfer function $T(s, \underline{x})$ has been used. Based upon the small gain theorem the $R S$ condition for an uncertain system subject to the unstructured multiplicative uncertainty is defined by (14) [24], [20]:

$$
R S:\left\|W_{T}(j \omega) T(j \omega, \underline{x})\right\|_{\infty} \leq 1 \Rightarrow\|T(j \omega, \underline{x})\|_{\infty} \leq \frac{1}{\left\|W_{T}(j \omega)\right\|_{\infty}} \text {, for } \omega \in\left[\omega_{\min }, \omega_{\max }\right]
$$

where $T(s, \underline{x})=G_{N}(s) K(s, \underline{x})\left[I+G_{N}(s) K(s, \underline{x})\right]^{-1}$ denotes the sensitivity function, which defines the transfer function from both inputs $\eta(s)$ and $r(s)$ to the output $y(s)$. Moreover $W_{T}(j \omega)$ presents any stable transfer function that majorates all possible uncertainties and satisfies the following condition (15):

$$
\sigma_{\max }\left[\Delta_{m}(j \omega)\right]=\sigma_{\max }\left[\left(G_{P}(j \omega)-G_{N}(j \omega)\right) G_{N}^{-1}(j \omega)\right]<<\sigma_{\max }\left[W_{T}(j \omega)\right]
$$

Design of Robust Fractional-Order PID Controller for DC Motor Using the Adjustable ... (Toufik Amieur) 
İn the next section, $W_{T}(s)$ will be assumed as the following fixed transfer function (16):

$$
W_{T}(s)=\frac{k_{T}\left(s+z_{1}\right)\left(s+z_{2}\right)}{\left(s+s_{1}\right)\left(s+s_{2}\right)}
$$

where its parameters are chosen similar to that given in [21].

\subsection{NP Condition}

During the design procedure, relatively fast responses, small overshoots and robustness against the model uncertainties can be assumed as suitable performances. Consequently, acquiring the $N P$ is a crucial factor that should be fulfilled by optimization. To ensure this goal, the sensitivity transfer function $S(s, \underline{x})$, has been used.

Noticing that, the sufficient small singular values $S(s, \underline{x})$ in specific frequency ranges can satisfy precise performance characteristics. Moreover, all these characteristics can be obtained by selecting the performance weight $W_{s}(s)$, which is used to shape the sensitivity function as follow (17) [24], [20]:

$$
N P:\left\|W_{S}(j \omega) S(j \omega, \underline{x})\right\|_{\infty} \leq 1 \Rightarrow\|S(j \omega, \underline{x})\|_{\infty} \leq \frac{1}{\left\|W_{S}(j \omega)\right\|_{\infty}} \text {, for } \omega \in\left[\omega_{\min }, \omega_{\max }\right]
$$

where $S(s)=\left[I+G_{N}(s) K(s, \underline{x})\right]^{-1}$ denotes the sensitivity function, which defines the transfer function from both inputs $d_{y}(s)$ and $r(s)$ to the output $e(s)$. In this paper the performance weight $W_{s}$ will be assumed as the adjustable transfer function that defined in (18). Its parameters are jointly optimized to those of the desired controller using some rules given that decribed later [25]. We get (18):

$$
W_{S}(s)=\frac{\omega_{B}+s / M_{s}}{\varepsilon_{S} \omega_{B}+s}
$$

where $\omega_{B}$ is the minimum bandwidth, which presents the straight-line approximation of $W_{S}(s)$ crosses the unity (i.e. $\left.0_{d b}\right), M_{S}$ is the minimum peak, which limits $\|S(s, x)\|_{\infty}$ and $\varepsilon_{S}$ is the steady-state error, which is fixed to the less value. İn this paper the good trade-off between $N P$ and $R S$ is constrained by the good selecting of the performance weight (18), in which the parameters $M_{S}$ and $\omega_{B}$ are given by optimization. So that these parameters are chosen according to the following rules.

i. Typically, the adjustable parameter $M_{S}$ is chosen to be sufficiently small. So that the pole of $1 / W_{S}$ is at least two decades above its zero. In general, it is required to have $M_{S}>>\varepsilon_{S}$.

ii. When the control objective is to improve the $N P$ margin, the general rule is to flat the curve of $\sigma_{\max }[S(j \omega, x)]$ as much as possible in high frequency. This goal is achieved by increasing $\omega_{B}$. However, increasing this frequency more than necessary deteriorates the $R S$ condition in highfrequency range.

Now, in the $S / T$ mixed sensitivity problem, both conditions (14) and (17) are combined and equation (18) is used to determine the following optimization problem (19) [25]:

$$
\min _{\underline{x} \in \chi}\left\|\begin{array}{l}
W_{S}(s, \underline{x}) \cdot S(s, \underline{x}) \\
W_{T}(s) \cdot T(s, \underline{x})
\end{array}\right\|_{\infty} \Leftrightarrow \min _{\underline{x} \in \chi}\left(\max _{\omega} \sigma_{\max }\left[\begin{array}{l}
W_{s}(s, \underline{x}) \cdot S(j \omega, \underline{x}) \\
W_{T}(s) \cdot T(j \omega, \underline{x})
\end{array}\right]\right) \text { where }\left\{\begin{array}{l}
\omega \in\left[\omega_{\min }, \omega_{\max }\right] \\
\chi: x \in\left[x_{\min }, x_{\max }\right]
\end{array}\right.
$$

Its solution is given using the fminimax function of the Matlab software, in which the new design parameter vector is defined by $x=[\underbrace{K_{p}, K_{i}, K_{d}, \tau, \lambda, \mu}_{K(s, \underline{x})}, \underbrace{M_{S}, \omega_{B}}_{W_{S}(s, \underline{\underline{x}})}]^{T}$. 


\section{SIMULATION RESULTS AND DISCUSSIONS}

\subsection{Motor Model}

The $D C$ machines are characterized by their simplicities and flexibilities. By means of various combinations of the shunt, series and the separately excited field windings, they can be designed to display a wide variety of volt-ampere or speed-torque characteristics for both dynamic and steady-state operation. The systems of $D C$ machines have been frequently used in many applications requiring a wide range of motor speeds and a precise output motor control. The diagram of typical $D C$ motor is shown by Figure 2.

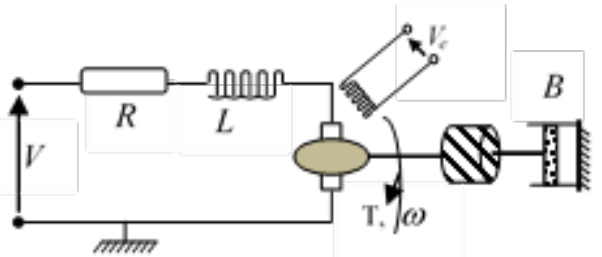

Figure 2. Schematic diagram of the $D C$ Motor

A well-known linear model of DC motor for the speed control system is shown in Figure 3.

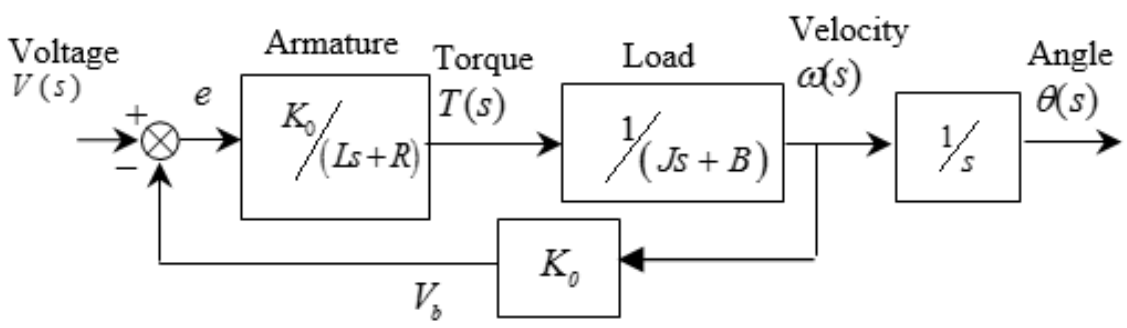

Figure 3. Bloc Diagram of DC Motor

According to Figure 3, the plant-model from the input voltage, $V(s)$ to the output velocity $\omega(s)$ is given by (20)

$$
\frac{\omega(s)}{V(s)}=\frac{K_{0}}{(L s+R)(J s+B)+K_{0}^{2}}
$$

Thus, the nominal plant-model from the angle $\theta(s)$ to the voltage $V(s)$ is defined as (21)

$$
G_{N}(s)=\frac{\theta(s)}{V(s)}=\frac{1}{s}\left[\frac{K_{0}}{(L s+R)(J s+B)+K_{0}^{2}}\right]
$$

The nominal values of $D C$ motor are summarized by Table1. We get

Table 1: DC Motor parameters

\begin{tabular}{ccc}
\hline Motor Parameters & Value & Unity \\
\hline$J$ & 0.02 & $\mathrm{~kg} . \mathrm{m}^{2} / \mathrm{s}^{2}$ \\
$B$ & 0.2 & $\mathrm{~N}-\mathrm{m} . \mathrm{s} / \mathrm{rad}$ \\
$K_{0}$ & 0.1 & $\mathrm{~N} . \mathrm{m} / \mathrm{A}$ \\
$R$ & 2 & $\mathrm{ohm}$ \\
$L$ & 0.5 & $\mathrm{H}$ \\
\hline
\end{tabular}


For the weighted-mixed sensitivity formulation problem, the frequency range $\left[\omega_{\min }, \omega_{\max }\right]=\left[10^{-4}, 10^{+4}\right]$ is used where the same fixed robustness weight of [21] is chosen. We get (22):

$$
W_{T}(s)=\frac{0.2619(s+17.38)(s+4.187)}{(s+21.26)(s+5.018)}
$$

In the next section, the adjustable weighting function $W_{S}(s, \underline{x})$ is defined by $W_{S}(s)=\frac{\omega_{B}+s / M_{s}}{10^{-4} \omega_{B}+s}$, where $1 \leq M_{s} \leq 1.5$ and $60 \leq \omega_{B} \leq 100$

For the FO-PID synthesis controller step, the lower and upper bounds that limit the design controller vector are chosen as $\left\{\begin{array}{l}1 \leq K_{p}, K_{i}, K_{d} \leq 300 \\ 10^{-6} \leq \tau \leq 10^{-2} \\ 10^{-4} \leq \lambda, \mu \leq 1\end{array}\right.$, where the fminimax function is initialized by the lower bound vector $x_{0}=[\underbrace{1,1,1,10^{-6}, 10^{-4}, 10^{-4}}_{K\left(s, \underline{x}_{0}\right)}, \underbrace{1,60}_{W_{S}\left(s, \underline{x}_{0}\right)}]^{T}$. The obtained solution of (19) yields also the following transfer functions

$$
\begin{aligned}
& \text { a) PFO-PID: } W_{S_{p}}\left(s, \underline{x}^{*}\right)=\frac{70.2270+s / 1.001229}{70.2270 \times 10^{-4}+s}, K\left(s, \underline{x}^{*}\right)=196.785+24.919 s^{-0.9631}+\frac{110.857 s^{0.9931}}{1+0.00032 s} \\
& \text { b) SFO-PID: } W_{S_{s}}\left(s, \underline{x}^{*}\right)=\frac{80.8870+s / 1.02386}{80.8870 \times 10^{-4}+s}, K\left(s, \underline{x}^{*}\right)=200.026\left(1+12.1843 s^{-0.103}\right) \cdot\left(1+\frac{0.5667 s^{0.994}}{1+0.00851 s}\right) \text {. }
\end{aligned}
$$

The obtained performances by the PFO-PID controller are compared with those given by the conventional integer $H_{\infty}$ controller. Its transfer function is given by the Hinflmi function of the Matlab environment which solves the weighted-mixed sensitivity problem based upon both weights $W_{S_{P}}\left(s, \underline{x}^{*}\right)$ and $W_{T}(s)$. Nevertheless, the performances of the SFO-PID controller are compared with those given by the conventional integer $H_{\infty}$ controller, in which both weights $W_{S_{s}}\left(s, \underline{x}^{*}\right)$ and $W_{T}(s)$ are used in the optimization problem. Finally, the obtained performances of previous controllers are compared with those given by the conventional integer PID controller, which its transfer function is given by $K(s)=124.358+14.338 s^{-1}+\frac{178.877 s}{1+0.001 . s}$.

Figure 4 compares the singular values plots of SFO-PID, conventional integer PID and conventional $H_{\infty}$ controllers in frequency domain. However, Figure 5 compares the singular values plots of PFO-PID, conventional integer PID and conventional $H_{\infty}$ controllers.

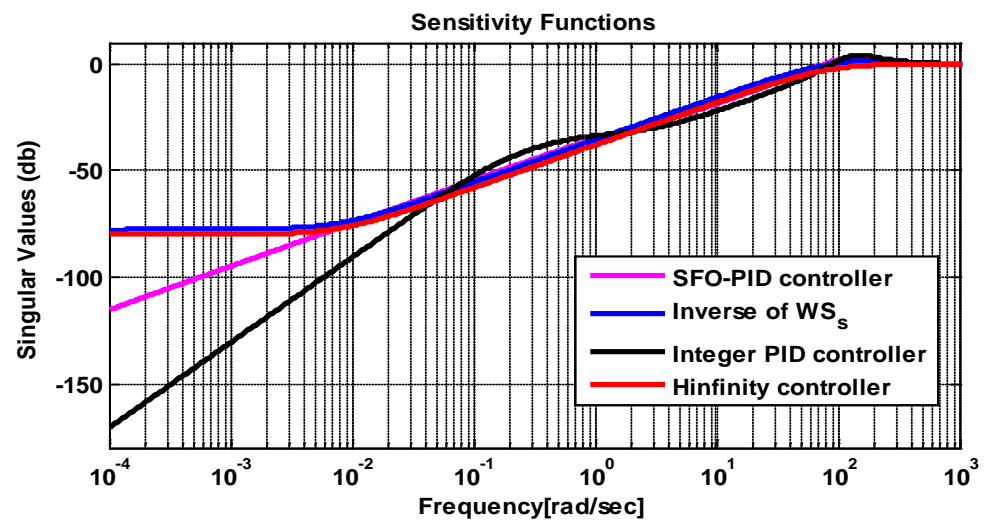

Figure 4. Nominal performance properties given by SFO-PID, integer PID and $H_{\infty}$ controllers 
According to figure 4, it can be seen that maximal singular values plot given by the integer PID controller exceed the upper bounds, $1 / \sigma_{\max }\left[W_{S_{s}}\left(j \omega, \underline{x}^{*}\right)\right]$ at some frequencies except, in frequency range $\omega \in[0.07,1.33]$ radians/seconds. This can be explained in the time domain by higher sensitivity to sensor noises. Noticing that the better NP margin is given when the maximum singular values plot of the sensitivity function are small as much as possible at low-frequency range. So that, the robust SFO-PID controller ensures the better margin then the $H_{\infty}$ controller. Furthermore, in low frequency range when $\omega \leq 0.005$ radians/seconds, the curve of $\sigma_{\max }\left[S\left(j \omega, x^{*}\right)\right]$ is below $-80 \mathrm{~dB}$, which means that the load disturbances are attenuated more than 10000 times at plant output.

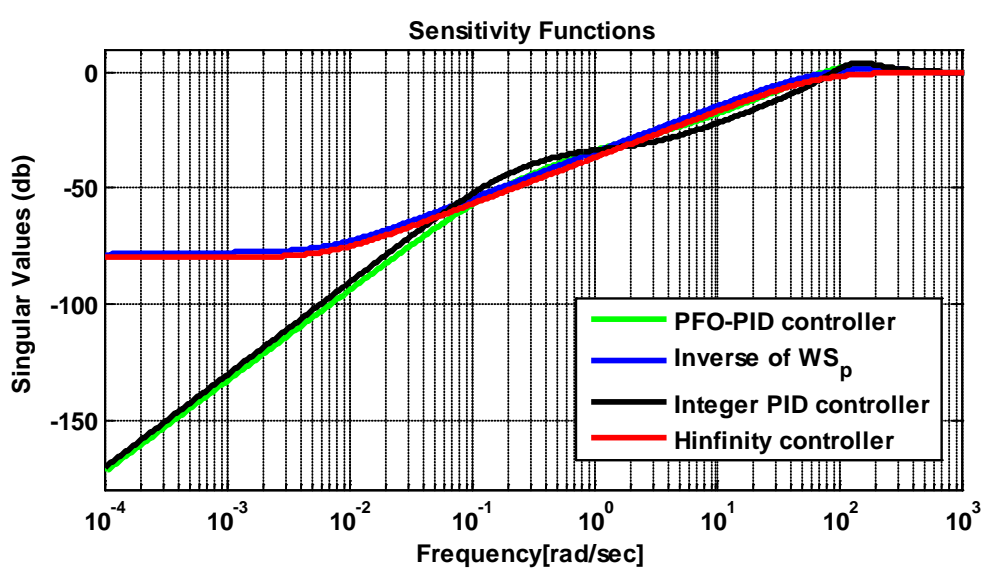

Figure 5. Nominal performance properties given by PFO-PID, integer PID and $H_{\infty}$ controllers

According to figure 5, the robust PFO-PID controller ensures the better NP margin than the one given by $H_{\infty}$ controller; integer PID controller and also the robust SFO-PID controller as shown in figure 6 . For the RS case, figure 7 compares the singular values plots of SFO-PID, conventional integer PID and conventional $H_{\infty}$ controllers in frequency domain. However, Figure 8 compares the singular values plots of PFO-PID, conventional integer PID and conventional $H_{\infty}$ controllers.

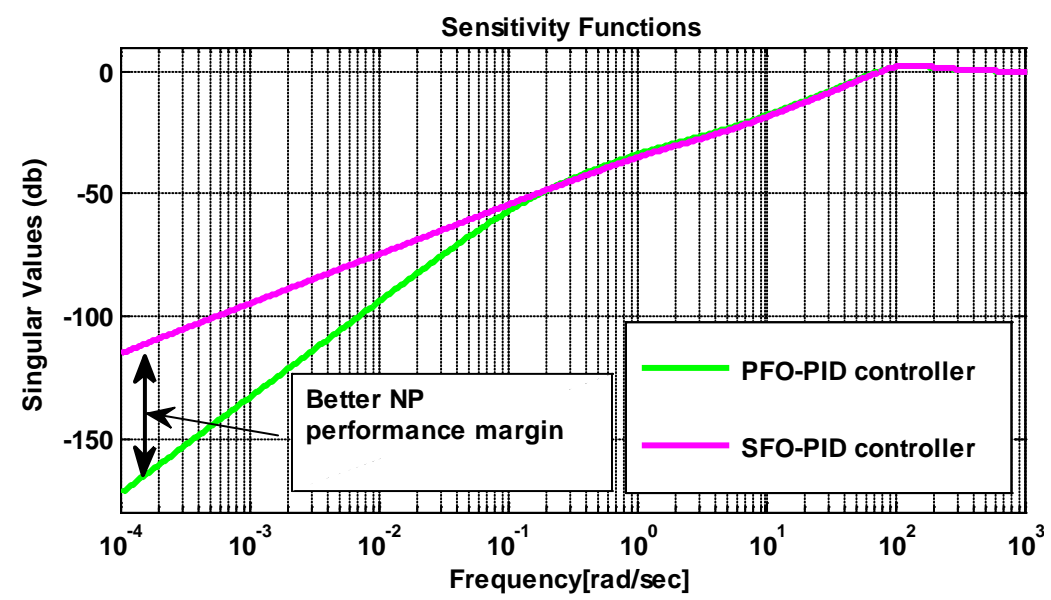

Figure 6. Nominal performance properties: PFO-PID vs SFO-PID

According to figures 7 and 8, it can be seen that the better $R S$ margin is given when the maximum singular values of complementary sensitivity matrix are small as much as possible at high frequency range. So that, the $H_{\infty}$ ensures the better NP margin compared with those given by integer PID, PFO-PID and SFO-PID controllers. 


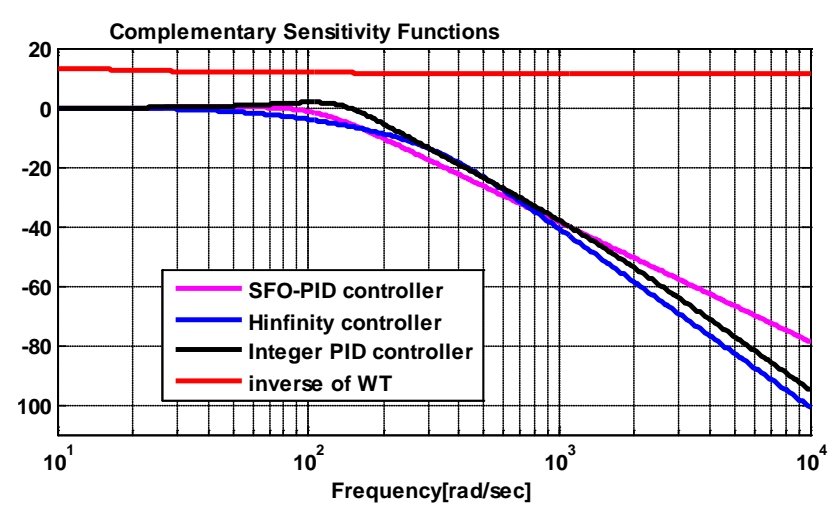

Figure 7. Robust stability properties given by SFO-PID, integer PID and $H_{\infty}$ controllers

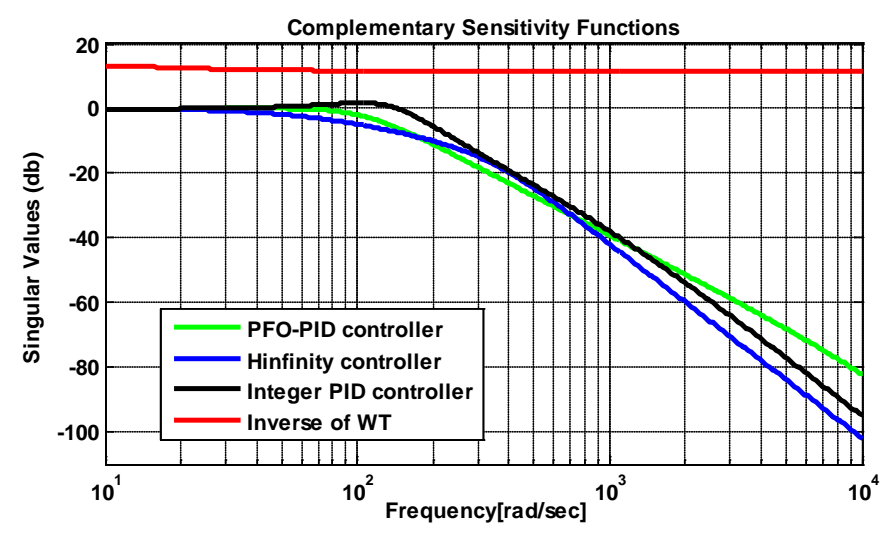

Figure 8. Robust stability properties given by PFO-PID, integer PID and $H_{\infty}$ controllers

According to figure 9, it is easy to see that the singular values plot, which is given by the PFO-PID controller is reduced at frequencies beyond the system bandwidth in order to secure robustness at high frequency range. Furthermore, for frequencies above $\omega=3200$ radians/seconds, this plot is below $-60 \mathrm{~dB}$ in which the sensor noises are suppressed more than 1000 time at the plant output. Consequently, the PFO-PID controller provides the better RS margin compared with the one given by the SFO-PID controller. To confirm the above results in time domain, the set-point reference that assumed a unit-step is used. Therefore, figure 10 shows the obtained tracking dynamic of the closed-loop system given by the $H_{\infty}$, integer $P I D$, robust $P F O$ $P I D$ and robust SFO-PID controllers. So that, the better tracking properties are ensured by the robust $P F O$ PID controller, which are characterized by the fast settling time with the reasonable overtaking.

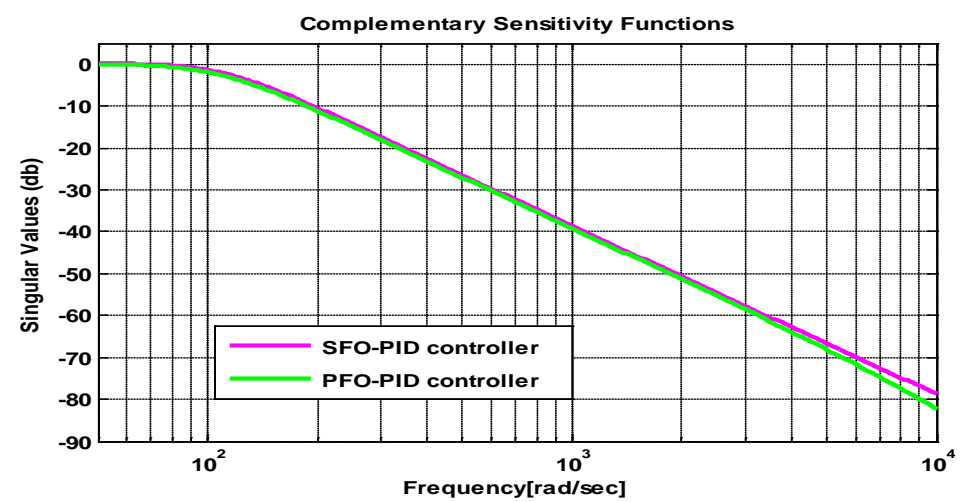

Figure 9. Robust stability properties: PFO-PID vs SFO-PID controllers 


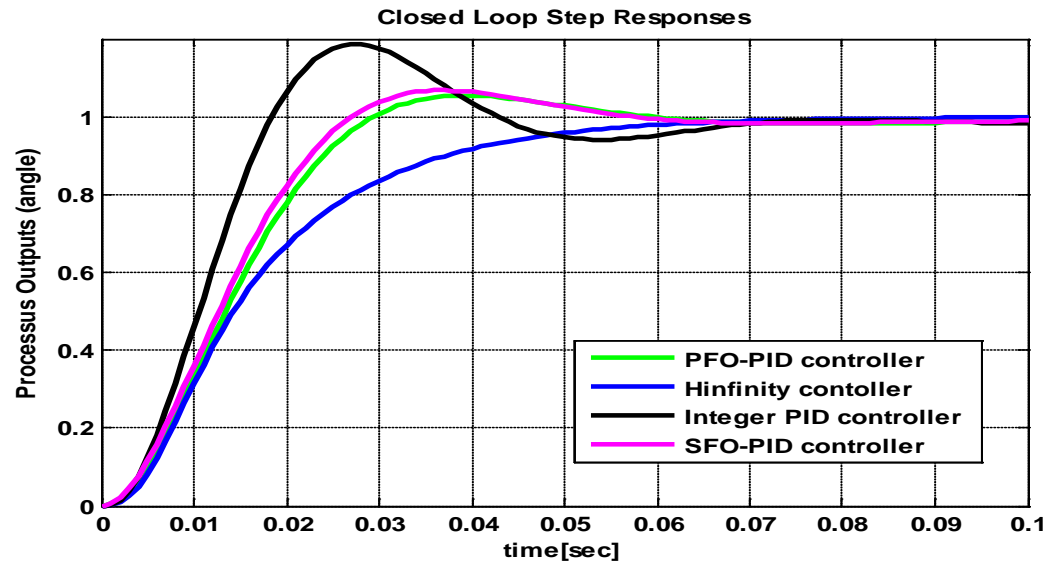

Figure 10. Tracking dynamic of the closed-loop system given by the $H_{\infty}$, integer PID, robust PFO-PID and robust SFO-PID controllers

\section{CONCLUSION}

In this paper, comparisons between two fractional controller-structures have been presented for $D C$ speed control motor. The design controllers have been achieved using the series and parallel fractional order PID configurations. Each controller has been designed with the automatic selection of corresponding performance weight. The parameters of both controller and weight have been determined from solving the weighted-mixed sensitivity problem by the fminimax function of the Matlab software. The obtained simulation results have been compared with those given by two conventional controllers. The obtained simulation results show the notable improvement that the proposed control strategy. However, it is also clear that further improvements in weighted-mixed sensitivity formulation step will require introducing the fractional weights to enhance the controller performances for the wide variation of the model parameters.

\section{ACKNOWLEDGEMENTS}

The authors would like to thank the Pervasive Artificial Intelligence PAI group of the informatics department of Fribourg-Switzerland for its valuable suggestions and comments which helped us to improve this paper. Special thanks to the prof. Béat Hirsbrunner and the Dr. Michèle Courant.

\section{REFERENCES}

[1] H. Zhang, X. Zhang, J. Wang, "Robust Gain-Scheduling Energy-to-Peak Control of Vehicle Lateral Dynamics Stabilization”, Veh. Syst. Dyn, Vol. 52, No. 3, pp. 309-340, 2014.

[2] H. Zhang, Y. Shi, J. Wang, “On Energy-to-Peak Filtering for Nonuniformly Sampled Nonlinear Systems: A Markovian Jump System Approach”, IEEE Trans. Fuzzy Syst. Vol. 22, No. 1, pp. 212 - 222, 2014.

[3] J. Kang, W.J. Meng, A. Abraham, and H.B. Liu, "An Adaptive PID Neural Network for Complex Nonlinear System”, Neurocomputing, 135, pp. 79 - 85, 2014.

[4] G.Q. Zeng, K.D. Lu, Y.X. Dai, Z.J. Zhang, M.R. Chen, C.W. Zheng, D. Wu, and W.W. Peng, "Binary-Coded Extremal Optimization for the Design of PID Controllers”, Neurocomputing, 138, pp. 180 - 188, 2014.

[5] G.Q. Zeng, J. Chen, M.R. Chen, Y.X. Dai, L.M. Li, K.D. Lu, and C.W. Zheng, "Design of Multivariable PID Controllers Using Real-Coded Population-Based Extremal Optimization”, Neurocomputing, 151, pp. 1343-1353, 2015.

[6] K. Glover, J. C. Doyle, "State-Space Formulae For All Stabilizing Controllers that Satisfy an $\mathrm{H}_{\infty}$ Norm Bound and Relations to Risk Sensitivity”. Sys. Cont. Letters, Vol. 11, pp. 167-172, 1988.

[7] A. A. Jalali, H. Golmohammad, "Line-of-Sight Stabilization by Robust $\mathrm{L}_{1}$ Controller Based on Linear Matrix Inequality (LMI) Approach”. Journal of Control Engineering and Applied Informatics, CEAI, Vol.15, No.1 pp. 6370, 2013.

[8] I. Podlubny, "Fractional-Order Systems and $P I^{\lambda} D^{v}$ Controller”. IEEE Transaction on automatic Control, Vol. 44, pp. 208-214, 1999.

[9] C.I. Muresan, S. Folea, G. Mois, and E.H. Dulf, "Development and Implementation of an FPGA Based Fractional Order Controller for a DC Motor”. Mechatronics, Vol. 23, pp. 798-804, 2013.

[10] J. Villagra, B. Vinagre, I. Tejado, "Data-driven Fractional PID Control: Application to DC Motors in Flexible Joints”. In: IFAC conference on advances in PID control. Brescia, Italy, 2012. 
[11] I. Pan, S. Das, “Chaotic Multi-objective Optimization Based Design of Fractional Order $P I^{\lambda} D^{\mu}$ Controller in AVR System”. Int J Electr Power Energy Syst, Vol. 43, pp. 393-407, 2012.

[12] I. Pan, S. Das, "Frequency Domain Design of Fractional Order PID Controller for AVR System Using Chaotic Multi-objective Optimization”. Int J Electr Power Energy Syst, Vol. 51, pp.106-118, 2013.

[13] M.A. Aboelela, M.F. Ahmed, H.T. Dorrah, "Design of Aerospace Control Systems Using Fractional PID Controller”. J Adv Res, Vol. 3, pp. 225-232, 2012.

[14] L. Jia, M. Xiuyun, L. Zaozhen. "Free Style Fractional Order Controller Design with PSO for Weapon System”. Energy Procedia, Vol. 13, pp. 7577-7582, 2011.

[15] R. Melício, V.M.F. Mendes, J.P.S. Catalão, "Fractional-order Control and Simulation of Wind Energy Systems with PMSG/Full-power Converter Topology”. Energy Convers Manage, Vol. 51, pp. 1250-1258, 2010.

[16] S. Das, I. Pan, S. Das, "Fractional Order Fuzzy Control of Nuclear Reactor Power with Thermal-Hydraulic Effects in the Presence of Random Network Induced Delay and Sensor Noise Having Long Range Dependence”. Energy Convers Manage, Vol. 68, pp. 200-218, 2013.

[17] Z.H. Chen, X. H. Yuan, B. Ji, P. T. Wang, and H. Tian, "Design of a Fractional Order PID Controller for Hydraulic Turbine Regulating System Using Chaotic Non-Dominated Sorting Genetic Algorithm II". Energy Convers Manage, Vol. 84, pp. 390-404, 2014.

[18] C. A. Monje, Y. Q. Chen, B. M. Vinagre, D. Xue, and V. Feliu, "Fractional-order Systems and Controls: Fundamentals and Applications, Advances in Industrial Control”. London: Springer Verlag, 2010.

[19] Y. Bensafia, and S. Ladaci, "Adaptive control with fractional order reference model”. International journal of sciences and techniques of automatic control \& computer engineering, Vol. 5, pp.1614-1623, 2011.

[20] C. Zhihuan, Y. Xiaohui, J. Bin, W. Pengtao, and T. Hao, "Design of a Fractional Order PID Controller for Hydraulic Turbine Regulating System Using Chaotic Non-Dominated Sorting Genetic Algorithm II”, Energy Conversion and Management, Vol.84, pp. 390-404, 2014.

[21] K. Zhou, J.C. Doyle, Essential of Robust Control, New Jersey: Prentice-Hall, 1998.

[22] M. Sedraoui, S. Gherbi, S. Abdelmalek, “A Robust Controller Based on Fractional Structure for MIMO Plant with Multiple Delays”. Cont. intel. Sys., Vol. 40, pp. 83-89, 2012.

[23] C. Belfedal, S. Gherbi, M. Sedraoui, S. Moreau, G. Champenois, T. Allaoui, and M. A. Denai, "Robust Control of Doubly Fed Induction Generator for Stand-Alone Applications”. Elsevier, Elec. Power Sys. Resear. Vol. 80, pp. 230-239, 2010.

[24] I. Maruta, T. Hyoung, T. Sugie, "Fixed-structure $H_{\infty}$ controller: A Meta-Heuristic Approach-Using Simple Constrained Particle Swarm Optimization”. Automatica, Vol. 45, pp. 553-559, 2009.

[25] H. Oloomi, B. Shafai, "Weight Selection in Mixed Sensitivity Robust Control for Improving the Sinusoidal Tracking Performance”. Conference on Decision and Control. 42nd IEEE 1, pp. 300-305, 2003. 